\title{
THE IMPORTANCE OF CONCENTRATION EFFECTS AT THE ELECTRODE SURFACE IN ANODIC STRIPPING VOLTAMMETRIC MEASUREMENTS OF COMPLEXATION OF METAL IONS AT NATURAL WATER CONCENTRATIONS
}

\author{
A. M. ALMEIDA MOTA ${ }^{a}$, J. BUFFLE*, S. P. KOUNAVES and M. L. SIMOES \\ GONCALVES ${ }^{a}$ \\ Department of Inorganic and Analytical Chemistry, University of Geneva, Sciences II, \\ 30, quai E. Ansermet, 1211 Geneva 4 (Switzerland)
}

(Received 1st November 1984)

\section{SUMMARY}

The influence of the ligand/metal ion concentration ratio on the shape, peak current and peak potential of curves obtained by anodic stripping voltammetry (a.s.v.) at the hanging mercury drop electrode is described, particularly with respect to the use of a.s.v. for speciation of metal ions at very low concentrations as is often found in natural waters. The lead(II)/triethylenetetramine system is used as a model of a fully labile reversible system. It is shown that the total metal ion concentration at the electrode surface $\left(C_{M}^{o}\right)$ during the stripping step may be much larger (30-300 times in typical conditions) than that in the bulk solution $\left(C_{M}\right)$, the exact value depending on the deposition time $t_{\mathrm{d}}$. Consequently, changes in the peak characteristics are observed when the ligand $/ \mathrm{metal}$ concentration ratio in the bulk of the solution, $C_{\mathrm{L}} / C_{\mathrm{M}}$, is less than 1000 . Semi-empirical equations, experimentally tested, are given, which enable $C_{M}^{9} / C_{M}$ to be estimated for a specified solution and a.s.v. conditions, which correct for the "surface concentration effect" when a.s.v. is used to measure complexation, and which describe the influence of the parameters such as stirring efficiency, radius of the mercury drop and $C_{\mathrm{L}} / C_{\mathrm{M}}$. The implications of the results are discussed for determinations of total metal ion in complex media, of speciation based on peak-potential shifts or stripping voltammetric curves, and of complexation capacity.

Anodic stripping voltammetry (a.s.v.) is widely used for the determination and speciation of trace metals in natural waters because the techniques can be applied directly, even at the extremely low concentrations found in these samples [1-3]. The a.s.v. procedure includes the deposition step, where the metal ion $M$ is reduced on the electrode surface during time $t_{\mathrm{d}}$, at a constant deposition potential, $E_{\mathrm{d}}$, and the stripping step, in which the metal is oxidized back into solution by scanning the potential towards more positive values. The deposition is, of course, a preconcentration step, thus the metal ion concentration at the electrode surface $\left(C_{M}^{0}\right)$ during the stripping step, is much larger than that in the bulk solution $\left(C_{M}\right)$.

aPresent address: Centro de Quimica Estrutural, Instituto Superior Tecnico, 1096 Lisboa, Portugal. 
Although this surface concentration effect is of no particular importance in the case of an uncomplexing medium, in a complexing medium it may drastically influence the ligand-to-metal concentration ratio, and so the concentration gradient of $\mathrm{M}$ at the electrode surface will be modified, as well as the shape of the current/potential curve [4-6]. Obviously, this effect will be negligible when the bulk ligand concentration $\left(C_{\mathrm{L}}\right)$ is so large that it is in great excess even at the electrode surface during the stripping step. Unfortunately, this is not the case for many ligands found in natural water systems.

Figure 1 indicates typical ranges of concentrations of the commonest metal ions determined by a.s.v., and of the most important dissolved aquatic ligands (more details are available elsewhere [7]). Except for a few inorganic

\section{sea waters}

\section{fresh water}
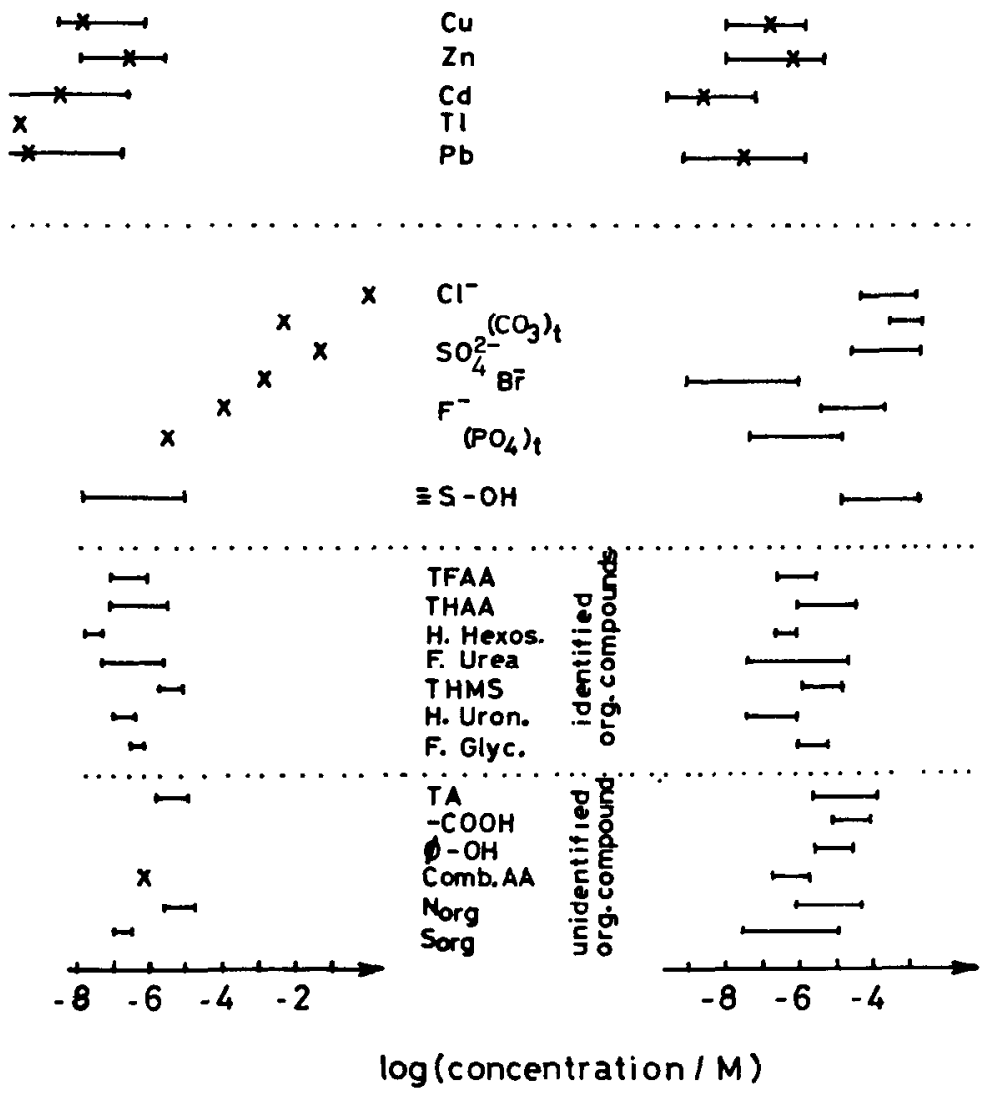

Fig. 1. Concentration ranges (-) and average values $(x)$ of metals and ligands in water. Concentration units (molarity) relate to monomers (identified compounds and Comb-AA), functional groups (TA, $-\mathrm{COOH}, \emptyset-\mathrm{OH}$ ), or donor atoms $\left(\mathrm{N}_{\mathrm{org}}, \mathrm{S}_{\text {org }}\right.$ ). H. Hexos., hydrolysable hexosamines; $\mathrm{H}$. Uron., hydrolysable uronic acids; $F$. glyc., free glycolic acid; $N_{\text {org }}, S_{\text {org }}$, organic $N$ and $S$; TFAA (THAA), total free (hydrolysable) amino acids, THMS, total hydrolysable monosaccharides; F. Urea, free urea. 
ligands such as $\mathrm{Cl}^{-}, \mathrm{HCO}_{3}^{-}$and $\mathrm{SO}_{4}^{2-}, C_{\mathrm{L}} / C_{\mathrm{M}} \approx 100$ for most of the ligands and $C_{\mathrm{L}} / C_{\mathrm{M}}$ may sometimes be as low as 10 . It is then very important to establish whether a ratio between 10 and 100 in the bulk solution is large enough to ensure an excess of ligand at the electrode surface. The aims of this paper are first to evaluate quantitatively the influence of $C_{\mathrm{L}} / C_{\mathrm{M}}$ on certain a.s.v. peak characteristics (current $\left(i_{\mathrm{p}}\right)$, potential $\left(E_{\mathrm{p}}\right)$, and shape), then to estimate the minimum ratio above which this effect is negligible, and finally to provide a procedure allowing one to correct for this effect when it is not negligible.

In studies of trace metal in natural waters, these aspects are particularly relevant in the following situations: (1) if the total concentration of $M$ is calculated from $i_{\mathrm{p}}$ vs. $C_{\mathrm{M}}$ calibration data, without complete elimination of the ligand in the medium; (2) if the stability constants of labile ML complexes are measured from the shift in $E_{\mathrm{p}}$ with $C_{\mathrm{L}}[8] ;$; (3) if complexation of $\mathrm{M}$ is evaluated by recording $i_{\mathrm{p}}$ vs. $E_{\mathrm{d}}$ curves (stripping polarography) [9-12]; and (4) if the complexation capacity and complexation constants are calculated from $i_{\mathrm{p}}$ vs. $C_{\mathrm{M}}$ standard addition curves $[6,13-15]$. The last three cases will be discussed here.

The above effect was tested by using lead(II) and triethylenetetramine (TETA). In the conditions used, this ligand forms a well defined 1:1 complex [16]. In order to test specifically the surface effects, the bulk composition of the solution was held constant. Hence, in most cases, the increase in $C_{M}^{0}$ was obtained by increasing $t_{\mathrm{d}}$ at constant $C_{\mathrm{M}}$, and the parameters $i_{\mathrm{p}}$ and $E_{\mathrm{p}}$ were used to follow the resulting effect of surface concentration. This effect was also investigated by using a medium-exchange system, in which the solution in the cell is changed between deposition and stripping.

\section{EXPERIMENTAL}

\section{Reagents and apparatus}

All reagents were of analytical grade (Merck) except for TETA which was of $99 \%$ purity (Fluka). Stock solutions were $0.1 \mathrm{M}$ potassium nitrate acidified to $\mathrm{pH} 5.5,1.00 \times 10^{-2} \mathrm{M}$ lead nitrate acidified to $\mathrm{pH} 3.0,4.90 \times 10^{-2} \mathrm{M}$ TETA neutralized to $\mathrm{pH} 8.0$ (concentration verified by an acid-base titration), and triethanolamine buffer solution at $\mathrm{pH} 7.5$ with a concentration 10 times that of TETA.

The precision of the $\mathrm{pH}$ measurements was $\pm 0.01 \mathrm{pH}$ unit (Metrohm $\mathrm{pH}$ meter E-603). Buffer solutions of $\mathrm{pH} 4.00$ and $\mathrm{pH} 7.00$ (Merck) were used for calibration.

A Tacussel PRG5 polarograph was used for all experiments. The reference electrode, to which all potentials are referred, was $\mathrm{Ag} / \mathrm{AgCl} / \mathrm{sat} . \mathrm{KCl} / 0.10 \mathrm{M}$ $\mathrm{KNO}_{3}$. The working electrode was a Metrohm (EA-290) hanging mercury drop electrode (HMDE), with a cone-shaped capillary end (the immersed length was $1.7 \mathrm{~cm}$; the outside (o.d.) and inside diameters (i.d.) were 4.55 and $0.1 \mathrm{~mm}$ respectively). Because the geometry of the electrochemical cell strongly influences the reproducibility of the deposition step, a special plexi- 
glas cell was built (Fig. 2, I) which would ensure reasonably reproducible stirring efficiency. Stirring was provided by a synchronous motor (Metrohm E-504), and a teflon-covered magnetic bar (4 mm diameter), the length of which $(1.5 \mathrm{~cm})$ was appropriate to the internal bottom diameter of the cell in order to prevent lateral movement. The bottom of the cell was conical, in order to prevent fallen mercury drops from impeding the rotation of the stirrer. The immersed parts of the platinum counter electrode $(1 \mathrm{~cm}$ long, $1 \mathrm{~mm}$ diameter) and the reference electrode bridge $(1 \mathrm{~cm}$ long, $3 \mathrm{~mm}$ diameter) were positioned about $0.7 \mathrm{~cm}$ above the endplane of the HMDE capillary. Purified nitrogen for degassing was introduced by means of a polyethylene capillary tube ( $1 \mathrm{~mm}$ o.d.).

The radius of the mercury drops $\left(r_{0}\right)$, was determined by weighing a fixed number of drops, as a function of the divisions indicated on the HMDE (for $2,5,10$ divisions, $r_{0}=0.032,0.044$ and $0.055 \pm 0.002 \mathrm{~cm}$, respectively).

The average thickness of the diffusion layer $(\bar{\delta})$ in the solution during the deposition step was evaluated by recording the current, $i_{\mathrm{d}}$, in an uncomplexing solution and at a sufficiently negative potential so that $C_{\mathrm{Pb}}^{0}=0$, and then by using the equation [17]: $\bar{i}_{\mathrm{d}}=n F S D_{\mathrm{Pb}} C_{\mathrm{Pb}} / \bar{\delta}$, where $\bar{i}_{\mathrm{d}}$ is the average value of $i_{\mathrm{d}}$ and is independent of time at sufficiently negative values of $E_{\mathrm{d}}$ (Fig. 3), $n=2, F=96494 C, S$ is the surface area of the $\mathrm{Hg}$ drop, $D_{\mathrm{Pb}}$ is the diffusion coefficient of $\mathrm{Pb}^{2+}$ in solution $\left(8.3 \times 10^{-6} \mathrm{~cm}^{2} \mathrm{~s}^{-1}\right)$ [18], and $C_{\mathrm{Pb}}$ is the total concentration of $\mathrm{Pb}^{2+}$ in solution $\left(10^{-4} \mathrm{M}\right)$.

By using a variable-speed motor, it was found that $\bar{\delta}$ increases with $(1 / \omega)^{1 / 2}$, where $\omega$ is the rotation speed of the stirrer. With the synchronous

(I)

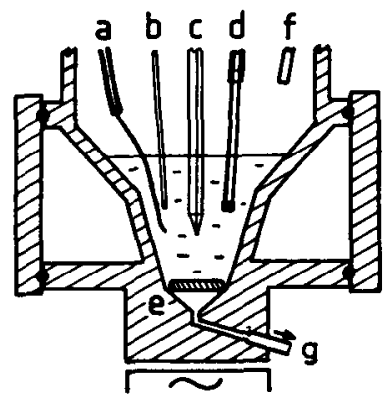

$1 \mathrm{~cm}$

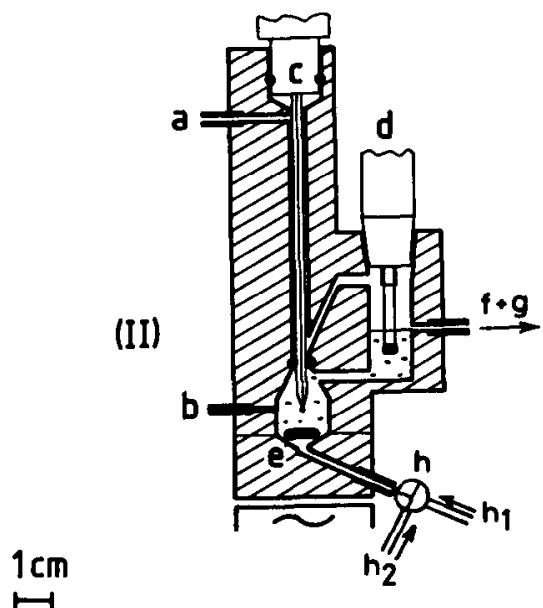

Fig. 2. Plexiglas cells for a.s.v.: (I) under classical conditions; (II) with medium exchange.

Parts: (a) nitrogen entrance; (b) Pt electrode; (c) HMDE; (d) reference electrode; (e) stirrer; (f) exit for nitrogen (and solution for cell II); (g) exit of solution (and $\mathrm{Hg}$ drops for cell $I)$; (h) three-way stopcock for entry of deposition solution $\left(h_{1}\right)$ and stripping solution $\left(h_{2}\right)$. 


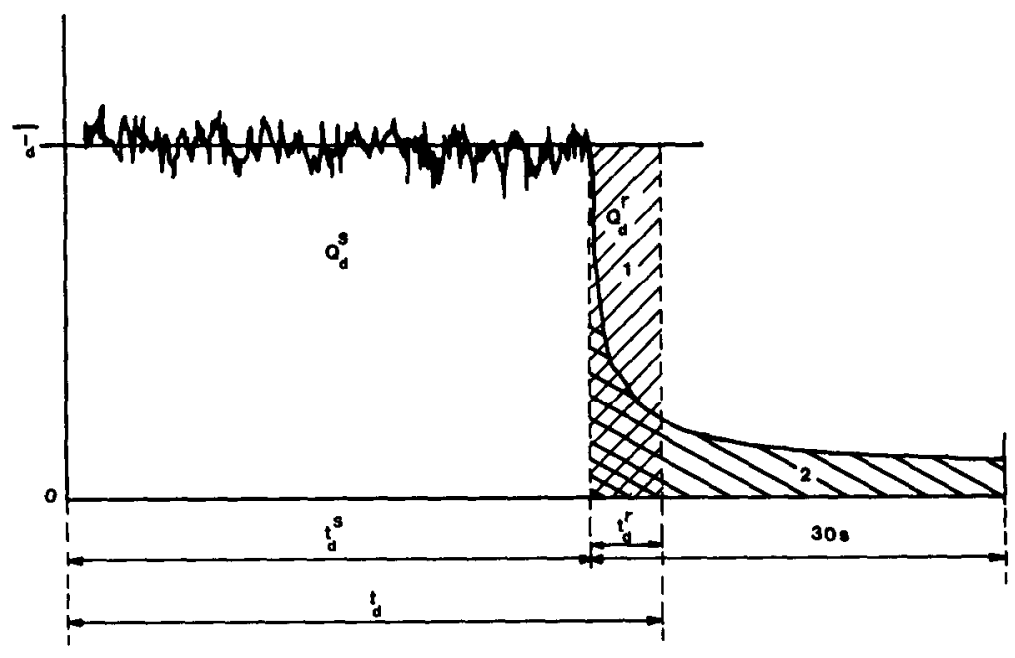

Fig. 3. The reduction current $i_{\mathrm{d}}$ as a function of deposition time $t_{\mathrm{d}} \cdot t_{\mathrm{d}}^{\mathrm{r}}$ is a fictitious time, such that $t_{\mathrm{d}}^{\mathrm{r}} \overline{i_{\mathrm{d}}}=Q_{\mathrm{d}}^{\mathrm{r}}=$ area $1=$ area $2=$ correction term to be applied to $t_{\mathrm{d}}^{\mathrm{s}}$ for evaluating the true value of $Q_{d}=Q_{d}^{s}+Q_{d}^{r}$.

motor, $\bar{\delta}$ was found to be $(1.2 \pm 0.1) \times 10^{-3} \mathrm{~cm}$. This value was measured before each experiment.

The polarographic cell used for medium exchange is shown in Fig. 2(II). Each solution was fully degassed before entering the polarographic cell (inlet h) and a flow of nitrogen was maintained in the cylindrical part around the capillary in order to prevent any penetration of air into the cell. Two reservoirs of degassed solution were connected to inlet $h$ : a "deposition" solution of $\mathrm{Pb}(\mathrm{II}) / \mathrm{TETA}\left(\mathrm{h}_{1}\right)$, and a "stripping" solution of $0.10 \mathrm{M}$ potassium nitrate at $\mathrm{pH} 5.5\left(\mathrm{~h}_{2}\right)$.

\section{Procedure}

All measurements were made at $25.0 \pm 0.1^{\circ} \mathrm{C}$, in $0.10 \mathrm{M}$ potassium nitrate. Experiments in the absence of TETA were done at pH 5.5 in order to prevent the hydrolysis of lead(II). In the presence of TETA, the solutions were buffered with triethanolamine at $\mathrm{pH}$ 8.0. It was checked that under these conditions the hydroxide and triethanolamine complexes of lead(II) should be negligible compared to the $\mathrm{Pb} / \mathrm{TETA}$ complex.

For all the deposition steps, a potential of $-0.700 \mathrm{~V}$ was applied, while stirring, for a time $t_{\mathrm{d}}^{\mathrm{s}}$. Then the stirring was stopped, and the solution was allowed to rest for $30 \mathrm{~s}$. The stripping step was then started by scanning the potential in the anodic direction at $20 \mathrm{mV} \mathrm{s}^{-1}$.

As can be seen in Fig. 3, the deposition that continues during the rest period, $t_{\mathrm{d}}^{\mathrm{x}}$, may be significant, especially when $t_{\mathrm{d}}^{8}$ is short. The total quantity of electricity corresponding to the deposition is given by $Q_{\mathrm{d}}=Q_{\mathrm{d}}^{s}+Q_{\mathrm{d}}^{\mathrm{x}}$, 
where $Q_{\mathrm{d}}^{\mathbf{s}}$ and $Q_{\mathrm{d}}^{\mathrm{r}}$ are the quantities of electricity corresponding to the stirring and rest periods, respectively. Dividing through by $i_{\mathrm{d}}$, the constant average deposition current, gives $Q_{\mathrm{d}} / \tilde{i}_{\mathrm{d}}=Q_{\mathrm{d}}^{\mathrm{s}} / \tilde{i}_{\mathrm{d}}+Q_{\mathrm{d}}^{\mathrm{r}} / \bar{i}_{\mathrm{d}}$ or $t_{\mathrm{d}}=t_{\mathrm{d}}^{\mathrm{s}}+t_{\mathrm{d}}^{\mathrm{r}}$. Here $t_{\mathrm{d}}$ is the "corrected" deposition time proportional to the total amount of lead deposited in the drop, and $t_{\mathrm{d}}^{\mathrm{r}}$ is the correction term. By recording curves similar to that in Fig. 3, for $C_{\mathrm{L}}=0$ and various concentrations of lead ions $\left(C_{\mathrm{Pb}}=\right.$ $10^{-4} \mathrm{M}, 10^{-6} \mathrm{M}$, and $\approx 10^{-8} \mathrm{M}$, corresponding to the concentration of lead(II) contaminations), it was observed that $t_{\mathrm{d}}^{\mathrm{r}}$ is independent of $C_{\mathrm{Pb}}$ and depends only on the mode of stirring. In the present conditions, it was found that $t_{\mathrm{d}}^{\mathrm{r}}=5 \mathrm{~s}$. This correction was applied in all cases and only $t_{\mathrm{d}}$ values are reported below. The same type of correction was applied in the case of medium exchange, but $t_{\mathrm{d}}^{\mathrm{r}}$ was found to be larger $(25 \mathrm{~s})$.

For the determination of the stability constants of the Pb/TETA complex, all $E_{\mathrm{p}}$ values (for cathodic sweep or a.s.v. techniques) in the presence of TETA, and the corresponding values in its absence, to which they are referred, were measured within the same set of experiments. All errors reported below are standard deviations, $\sigma$, defined as $\sigma=\left[\Sigma_{l}\left(x_{1}-x\right)^{2} /(N-1)\right]^{1 / 2}$, where $N$ is the number of determinations.

\section{Electrochemical characteristics of the lead(II)/TETA system}

The reported values of the acid-base constants of TETA and of its stability constant with lead(II), are given in Table 1 . Cathodic sweep voltammetry was used to test the electrochemical behaviour of the $\mathrm{Pb}$ (II)/TETA system with $C_{\mathrm{Pb}}=10^{-5} \mathrm{M}, C_{\mathrm{L}}=9.1 \times 10^{-3} \mathrm{M}$ and $\mathrm{pH} 8.02$. According to the usual criteria [19], $\left(E_{\mathrm{p}}-E_{\mathrm{p} / 2}=28 \mathrm{mV}\right.$, and independence of $E_{\mathrm{p}}, i_{\mathrm{p}} / v^{1 / 2}$ and $E_{\mathrm{p}}-E_{\mathrm{p} / 2}$ from the scan rate $v, E_{\mathrm{p} / 2}$ being the potential corresponding to $i_{\mathrm{p} / 2}$ ) it behaves as a labile reversible system, in the range $10<v<40 \mathrm{mV} \mathrm{s}^{-1}$.

For both a.s.v. and cathodic sweep voltammetry, the ratio of the peak currents, in the absence ( $\mathrm{pH} \mathrm{5.5)}$ and presence $\left(\mathrm{pH} \mathrm{8)}\right.$ of TETA, $i_{\mathrm{p}}^{\text {nc }} / i_{\mathrm{p}}^{\mathrm{c}}$,

\section{TABLE 1}

Characteristics of the lead(II)/TETA system ${ }^{a}$

\begin{tabular}{lllll}
\hline Reaction & $T\left({ }^{\circ} \mathrm{C}\right)$ & Constant & $\begin{array}{l}\text { Logarithm of } \\
\text { stability constant }\end{array}$ & Ref. \\
\hline $\mathrm{L}+\mathrm{H} \rightleftharpoons \mathrm{LH}$ & 25 & $\beta_{\mathrm{H}}^{\mathrm{H}}$ & 9.74 & 16 \\
$\mathrm{~L}+\mathrm{2H} \rightleftharpoons \mathrm{LH}$ & 25 & $\beta_{2}^{\mathrm{H}}$ & 18.82 & 16 \\
$\mathrm{~L}+\mathrm{Pb} \rightleftharpoons \mathrm{PbL}$ & 20 & $\beta$ & 10.4 & 16 \\
$\mathrm{~L}+\mathrm{Pb} \rightleftharpoons \mathrm{PbL}$ & 25 & $\beta$ & $10.18 \pm 0.03^{\mathrm{b}}$ & This work \\
\hline
\end{tabular}

TETA $=\mathrm{L}=\mathrm{NH}_{2}-\mathrm{CH}_{2}-\mathrm{CH}_{2}-\mathrm{NH}-\mathrm{CH}_{2}-\mathrm{CH}_{2}-\mathrm{NH}-\mathrm{CH}_{2}-\mathrm{CH}_{2}-\mathrm{NH}_{2}$. All data are for an ionic strength of $0.1 \mathrm{M}$. ${ }^{\mathrm{b}}$ Average of four determinations by differential-pulse cathodic sweep voltammetry with $C_{\mathrm{Pb}}=2 \times 10^{-5} \mathrm{M}$ and $C_{\mathrm{L}}$ varying between $0.98 \times 10^{-3}$ and $1.95 \times 10^{-2} \mathrm{M}$. 
were found to be $1.00 \pm 0.05$, and were independent of the values of $C_{\mathrm{Pb}}$ and $C_{\mathrm{L}}$, provided that TETA was in large excess ( $>30$ for cathodic sweep voltammetry and $>1000$ for a.s.v.). This result implies that $D_{\mathrm{PbL}}=D_{\mathrm{Pb}}$. Consequently, the shifts in $E_{\mathfrak{p}}$ in cathodic sweep voltammetry and in differential-pulse cathodic sweep voltammetry were used, as a further test of the electrochemical behaviour of the system, to compute the stability constant of the $\mathrm{Pb}$ (II)/TETA complex, according to Eqns. 2 and 3 (see below). The value shown in Table 1 is slightly smaller than the literature value, which may be due to the difference in temperature. In any case, considering the small amount of data available in the literature, the correspondence of the data in Table 1 was considered as satisfactory. In particular, the good agreement between the cathodic sweep voltammetry and the differential-pulse results, both for $i_{\mathrm{p}}^{\mathrm{nc}} / i_{\mathrm{p}}^{\mathrm{c}}$ and $E_{\mathrm{p}}^{\mathrm{nc}}-E_{\mathrm{p}}^{\mathrm{c}}$, is a further confirmation of the lability of the complexation reaction. The suffixes nc and $\mathrm{c}$ stand for noncomplexing $\left(C_{\mathrm{L}}=0\right)$ and complexing $\left(C_{\mathrm{L}} \neq 0\right)$ media respectively.

\section{THEORY, DEFINITIONS AND QUALITATIVE DESCRIPTION}

Discriminating between complexation at the electrode surface and in the bulk solution

For the lead(II)/TETA system described above, the degree of complexation of lead(II) in the bulk solution is given [20] by

$\alpha=C_{\mathrm{Pb}} /\left[\mathrm{Pb}^{2+}\right]=1+\beta[\mathrm{L}]=1+\beta\left(C_{\mathrm{L}}-[\mathrm{PbL}]\right) / \alpha_{\mathrm{H}}$

where $\alpha_{\mathrm{H}}=1+\beta_{1}^{\mathrm{H}}[\mathrm{H}]+\beta_{2}^{\mathrm{H}}[\mathrm{H}]^{2} ; C_{\mathrm{Pb}}$ and $C_{\mathrm{L}}$ are the total concentrations of lead(II) and TETA, and $\left[\mathrm{Pb}^{2+}\right],[\mathrm{L}]$ and $[\mathrm{H}]$ are the free metal ion, ligand and proton concentrations. When a.s.v. is used to quantify lead(II) in this system, the resulting curve depends on the degree of complexation of lead(II) at the electrode surface, both during the deposition step $\left(\alpha_{d}^{0}\right)$, and the stripping step $\left(\alpha_{s}^{0}\right)$. Therefore, it is easy to compute the value of $\alpha$ from the characteristic parameters of the peak, $E_{\mathrm{p}}$ and $i_{\mathrm{p}}$, only when $\alpha=\alpha_{\mathrm{d}}^{0}=\alpha_{\mathrm{s}}^{0}$. As will be shown below, this is valid when $C_{\mathrm{L}} \gg C_{\mathrm{M}}$. Then Eqn. 1 reduces to

$\alpha=1+\beta C_{\mathrm{L}} / \alpha_{\mathrm{H}}$

In such a case, for a labile, reversibly reduced system like $\mathrm{Pb}$ (II)/TETA, the DeFord and Hume method [21, 22], initially developed for d.c. polarography, can be applied to the a.s.v. technique

$\ln \alpha=(n F / R T)\left(E_{\mathrm{p}}^{\mathrm{nc}}-E_{\mathrm{p}}^{\mathrm{c}}\right)+\ln \left(i_{\mathrm{p}}^{\mathrm{nc}} / i_{\mathrm{p}}^{\mathbf{c}}\right)$

In the particular case of the lead(II)/TETA system, it was observed that $i_{\mathbf{p}}^{\text {nc }}=i_{\mathrm{p}}^{\mathrm{c}}$ so that the second term in Eqn. 3 vanishes. By combining Eqns. 2 and $3, \beta$ can easily be calculated, when $\alpha$ is a measured parameter.

Condition for $\alpha=\alpha_{\mathrm{d}}^{0}$ during the deposition step

The reduction of complexes without an excess of ligand in d.c. polarography has been discussed in detail [22]. For the reduction of metal complexes 
a) Deposition step

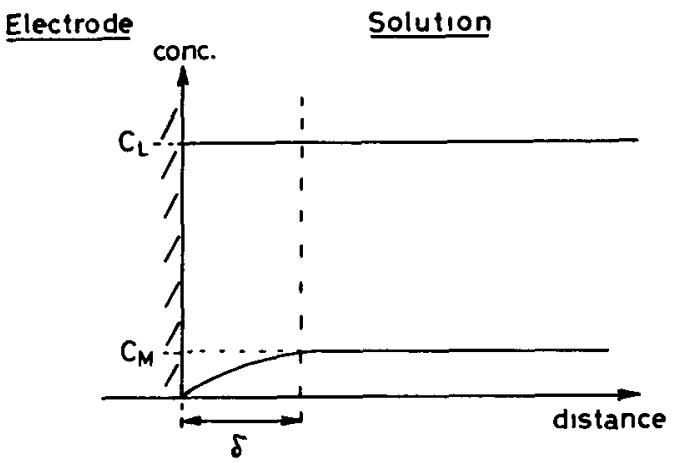

b) Stripping step

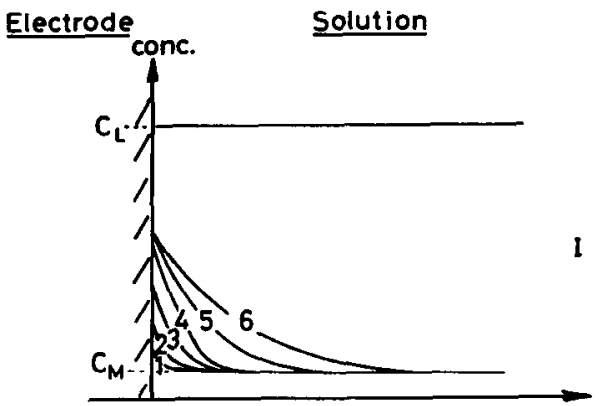

Fig. 4. Concentration gradients at the electrode surface during: (a) the reduction of $M$; (b) the stripping step for (I) various potentials, $E(1)<E(2)<E(3)<\ldots<E(6)$, at constant $t_{\mathrm{d}}$, and (II) various $t_{\mathrm{d}}, t_{\mathrm{d}}(1)<t_{\mathrm{d}}(2)<\ldots<t_{\mathrm{d}}(4)$, at the peak potential. $C_{\mathrm{L}}=$ $[\mathrm{PbL}]+[\mathrm{L}]+\Sigma_{l}\left[\mathrm{LH}_{l}\right]$.

to the metallic state, the consumption of $\mathrm{M}$ and the resulting liberation of $\mathrm{L}$ at the electrode surface (Fig. 4a), lead to the condition $\alpha_{\mathrm{d}}^{0}>\alpha$. Obviously, the difference between $\alpha_{\mathrm{d}}^{0}$ and $\alpha$ increases when going from the foot of the wave to the plateau. In d.c. polarography this results in a deformation of the reduction wave, and the same effect may be expected when constructing "pseudo polarograms" by stripping polarography.

However, if $C_{\mathrm{L}}$ is sufficiently large to fulfil the necessary condition for application of Eqn. 2 (e.g., $C_{\mathrm{L}} / C_{\mathrm{M}}>20$ ), it will be even larger at the electrode surface; thus one can estimate that the difference between $\alpha_{\mathbf{d}}^{0}$ and $\alpha$ is $\leqslant 5 \%$. It is maximal ( $5 \%)$ on the plateau of the wave.

\section{Conditions for $\alpha=\alpha_{\mathrm{s}}^{0}$ during the stripping step}

In contrast to the deposition step, the metal ion concentration during the stripping step is larger at the electrode surface than in the bulk solution (Fig. 4b). Consequently, one can expect that $\alpha_{\mathrm{s}}^{0}<\alpha$. The size of the difference between these two parameters depends on the surface concentration of M liberated during the stripping step. This was computed theoretically [5] 
for reversible systems in the absence of ligands for mercury film and hanging drop electrodes. For the HMDE, when $E_{d}$ is chosen on the plateau of the reduction wave, and the scan rate of the stripping step is large enough $\left(>5 \mathrm{mV} \mathrm{s}^{-1}\right.$ ) so that the bulk concentration of $\mathrm{M}^{\circ}$ in the mercury drop is not changed during the stripping, it was shown that at the peak potential, $E_{p}$

$C_{\mathrm{M}}^{0} / C_{\mathrm{M}}=(1 / 0.44)\left(D_{\mathrm{Ox}} D_{\mathrm{R}}\right)^{1 / 2} t_{\mathrm{d}} / r_{0} \delta$

where $D_{\mathrm{Ox}}$ and $D_{\mathrm{R}}$ are the diffusion coefficients of the metal ion in solution and of the metal in mercury, respectively. It should be noted that $C_{\mathrm{M}}^{0} / C_{\mathrm{M}}$ is independent of $v$, in contrast to $i_{\mathrm{p}}$ which increases with $v^{1 / 2}$ [18]. From Eqn. 4, using typical parameter values and with $t_{\mathrm{d}}=3 \mathrm{~min}$, it is possible to estimate that $C_{M}^{0} / C_{M} \approx 50$, i.e., surface concentrations much larger than bulk ones may be reached during the stripping step. Figure 4(b) shows the concentration gradients of $\mathrm{M}$ and $\mathrm{L}$ at $E_{\mathrm{p}}$, when the stripping occurs in the presence of an "excess" of complexing agent: (e.g., $C_{\mathrm{L}} / C_{\mathrm{M}}=30$ ): for a short $t_{\mathrm{d}}, C_{\mathrm{L}}^{0} \approx C_{\mathrm{L}}$ is still much larger than $C_{\mathrm{M}}^{0}$ and $\alpha_{\mathrm{s}}^{0}=\alpha$. However, for large $t_{\mathrm{d}}, C_{\mathrm{M}}^{0}$ may become larger than $C_{\mathrm{L}}^{0}$ so that $\alpha_{\mathrm{s}}^{0} \leqslant \alpha$. From the above estimate of $C_{\mathrm{M}}^{0} /$ $C_{\mathrm{M}}$, it may be expected that $C_{\mathrm{L}} / C_{\mathrm{M}}>1000$ is needed in the bulk solution, in order to ensure that $C_{\mathrm{L}}^{0} / C_{\mathrm{M}}^{0}>20$ at the electrode surface (for $t_{\mathrm{d}}=3 \mathrm{~min}$ ).

A qualitative description of the shape of the a.s.v. curves can be understood from Figs. $4(\mathrm{~b}, \mathrm{I})$ and 5 . Figure $4(\mathrm{~b}, \mathrm{I})$ shows the concentration gradients of $M$ at different potentials during the stripping step for a given value of $t_{\mathrm{d}}$. The surface concentration of $M$ increases in the potential range corresponding to the rising portion of the a.s.v. curve, and remains constant from then on (cf. [5]). When $t_{\mathrm{d}}$ is small enough, $C_{\mathrm{L}}^{0} / C_{\mathrm{M}}^{0} \approx C_{\mathrm{L}} / C_{\mathrm{M}}$ for all potentials, and a peak with a normal shape is obtained. For intermediate values of $t_{\mathrm{d}}, C_{\mathrm{Z}}>$ $C_{\mathrm{M}}^{0}$ for all potentials, but $C_{\mathrm{L}}^{0} / C_{\mathrm{M}}^{0}$ is much smaller at $E_{\mathrm{p}}$ than at the foot of the peak (see Fig. $4 \mathrm{~b}, \mathrm{I}$ ). As a consequence, $\alpha_{\mathrm{s}}^{0}$ decreases all along the peak, and the resulting peak is broadened (Fig. 5, curve 1).

Finally, for still larger values of $t_{\mathrm{d}}$, $\mathrm{L}$ becomes fully saturated at the electrode surface $\left(C_{\mathrm{L}}^{0} \approx C_{\mathrm{M}}^{0}\right)$ at a potential, $E_{\mathrm{sat}}<E_{\mathrm{p}}$. For $E>E_{\text {sat }}, \alpha_{\mathrm{s}}^{0} \approx 1$, thus the oxidation is greatly decreased, until the potential reaches a value corresponding to that for the oxidation in an uncomplexing medium. In such cases, a shoulder appears in the a.s.v. curves (Fig. 5, curves 2 and 3) for systems such that $E_{\mathrm{p}}^{\mathrm{nc}}-E_{\mathrm{p}}^{\mathrm{c}}(0)$ is large enough $\left(E_{\mathrm{p}}^{\mathrm{c}}(0)\right.$ is the value of $E_{\mathrm{p}}^{\mathrm{c}}$ at $t_{\mathrm{d}}=0$, see Fig. 6). The height of the shoulder, $i_{\mathrm{sh}}$, depends mainly on $C_{\mathrm{L}}$, and does not vary when $t_{\mathrm{d}}$ increases (Fig. 6). Hence, for extremely large values of $t_{\mathrm{d}}$, the largest part of $\mathrm{M}^{0}$ is reoxidized as free $\mathrm{M}^{2+}$, giving rise to the corresponding a.s.v. peak, and the relative contribution of the shoulder to the total current becomes increasingly smaller, although its absolute value remains constant.

The influence of $t_{\mathrm{d}}$ on the a.s.v. peak parameters, $E_{\mathrm{p}}^{\mathrm{c}}, i_{\mathrm{p}}^{\mathrm{c}}$ and $i_{\mathrm{sh}}$, as described above, is shown in Fig. 6. 


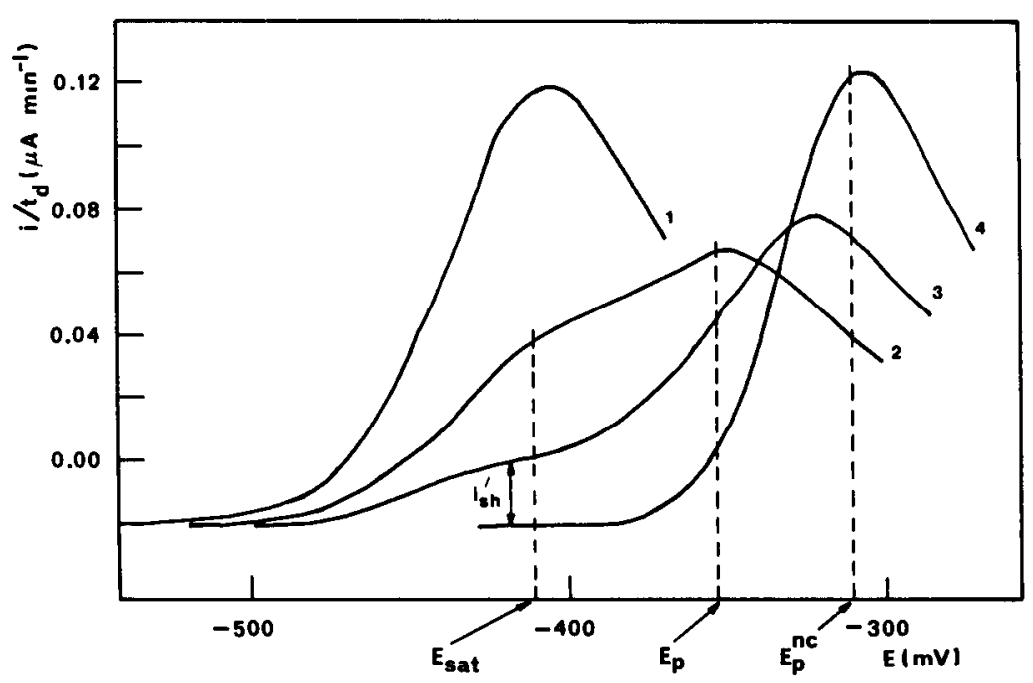

Fig. 5. "Normalized" anodic stripping voltammograms $\left(i / t_{\mathrm{d}}\right.$ vs. potential) of lead ion $\left(C_{\mathrm{M}}\right.$ $\left.=1.00 \times 10^{-6} \mathrm{M}\right):(1-3)$ in presence of TETA $\left(C_{\mathrm{L}}=0.98 \times 10^{-4} \mathrm{M}\right)$ for $t_{\mathrm{d}}=1,7$ and $25 \mathrm{~min}$, respectively; (4) in the absence of TETA. Other experimental conditions: $10^{-3} \mathrm{M}$ triethanolamine, $\mathrm{pH} 8.02, \mu=0.10 \mathrm{M}\left(\mathrm{KNO}_{3}\right) ; T=25.0^{\circ} \mathrm{C} ; r_{0}=0.032 \mathrm{~cm} ; v=20 \mathrm{mV} \mathrm{s}^{-1}$; $\bar{\delta}=1.2 \times 10^{-3} \mathrm{~cm} . E_{\text {sat }}$ and $E_{\mathrm{p}}$ are given for curve 2 (see text) $i_{\mathrm{sh}}^{\prime}=i_{\mathrm{sh}} / t_{\mathrm{d}}$.
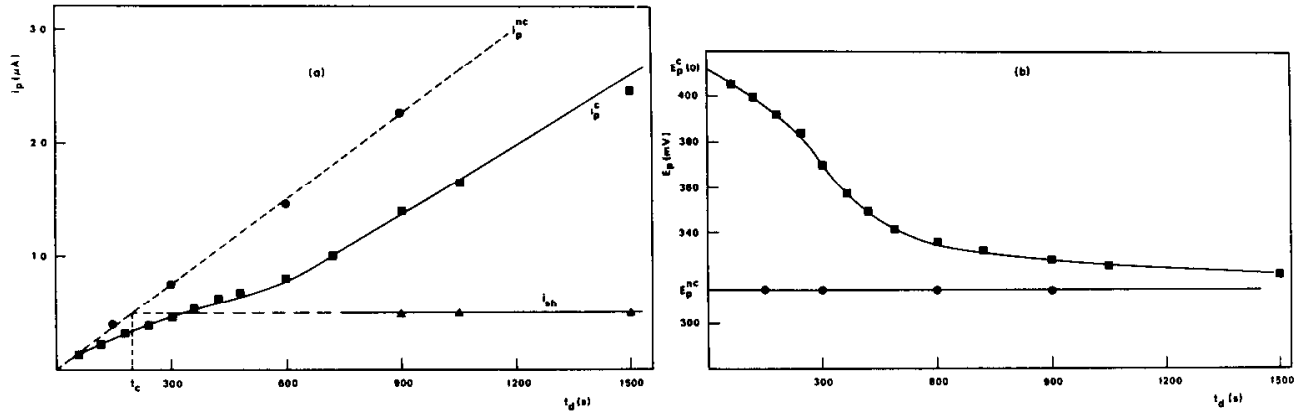

Fig. 6. Changes in the characteristic parameters of a.s.v. peaks $\left(E_{\mathrm{p}}, i_{\mathrm{p}}\right)$ as a function of $t_{\mathrm{d}}$ : (•) points corresponding to the a.s.v. peak of the metal in the absence of the ligand; (-) points corresponding to the a.s.v. peak of the metal in the presence of the ligand; (4) points corresponding to the a.s.v. peak shoulder height $i_{\text {sh }}$. Experimental conditions as in Fig. 5.

\section{Definition of critical deposition time}

From the above description, it is clear that the ratio $C_{\mathrm{M}}^{0} / C_{\mathrm{M}}$ is a critical factor for correct interpretation of the a.s.v. parameters $\left(E_{\mathrm{p}}\right.$ or $\left.i_{\mathrm{p}}\right)$ obtained in any complexing medium. At present, there is no theoretical relationship which allows one to predict the values of $i_{\mathrm{p}}, E_{\mathrm{p}}$, or $C_{\mathrm{M}}^{0} / C_{\mathrm{M}}$, in conditions where there is no excess of ligand at the electrode surface during the stripping step. 
The usefulness of Eqn. 4 for this purpose was therefore tested in the following part of this work. Although it is rigorously valid only in the absence of ligands, it may be expected that the influence of each of its parameters should be similar in complexing media and that it could be used as a semiempirical equation for analytical prediction. In this respect, the lead(II)/ TETA system is very suitable, because it was shown that the diffusion coefficients of the free and complexed forms of lead(II) are equal.

In order to test Eqn. 4 rigorously, it would be necessary to measure $C_{M}^{0}$ as a function of $t_{\mathrm{d}}$. This is not possible, and so use was made of the fact that a shoulder appears in the a.s.v. curves when $C_{\mathrm{M}}^{0} \approx C_{\mathrm{L}}$ and remains constant for $C_{M}^{0}>C_{L}$, even for a very large $t_{d}$ when $C_{M}^{0} \gg C_{L}$ (Figs. 5 and 6). The height of this shoulder $\left(i_{\mathrm{sh}}\right)$ was then used to define a critical deposition time, $t_{\mathrm{c}}$ (as shown in Fig. 6), corresponding to the minimum value of $t_{\mathrm{d}}$ for which TETA is considered to be saturated at the electrode surface. Then one has $C_{\mathrm{L}}^{0}=C_{\mathrm{L}}$ $=C_{\mathrm{M}}^{0}$, and

$$
t_{\mathrm{c}}=K\left(r_{\mathrm{o}} \delta / D_{\mathrm{Ox}} D_{\mathrm{R}}\right)\left(C_{\mathrm{L}} / C_{\mathrm{M}}\right)
$$

The experimental measurement of $t_{\mathrm{c}}$ was chosen so that the condition $C_{\mathrm{L}}^{0}=$ $C_{\mathrm{L}}=C_{\mathrm{M}}^{0}$ was reasonably fulfilled. Even though this may not be rigorously true, $t_{\mathrm{c}}$ can be considered as a useful semiempirical parameter for testing the influence of the factors in Eqn. 5. Because Eqn. 4 was not deduced for the presence of ligands, the numerical constant $K$ could be different from 0.44 , but its order of magnitude should be similar. The influence of the selected parameter on the interpretation of the results was tested by defining another critical deposition time, $t_{\mathrm{c}}(E)$, from the $E_{\mathrm{p}}^{\mathrm{c}}$ vs. $t_{\mathrm{d}}$ curve (Fig. 6). This curve may be considered as a compleximetric titration curve of $L$ by $M$ inside the diffusion layer, the potential jump corresponding to the end-point of the titration. Because the break in this curve is always more clearly measurable than the inflection point, it was used to define $t_{\mathrm{c}}(E)$ (Fig. 6). In all cases tested, the effects of the parameters of Eqn. 4 on $t_{\mathrm{c}}$ and $t_{\mathrm{c}}(E)$ were similar. However, the measurement of $t_{\mathrm{c}}(E)$ was less precise and only the results obtained with $t_{\mathrm{c}}$ are reported here.

\section{RESULTS}

Influence of $\mathrm{r}_{0}, \bar{\delta}$, and $\mathrm{C}_{L} / \mathrm{C}_{P b}$

The changes in $t_{\mathrm{c}}$ with $r_{0}, \bar{\delta}$, and $C_{\mathrm{L}} / C_{\mathrm{M}}$ are given in Table 2 and Fig. 7 . As predicted by Eqn. 5, a linear relationship is observed between $t_{\mathrm{c}}$ and $r_{0} \bar{\delta}\left(C_{\mathrm{L}} / C_{\mathrm{M}}\right)$. From the slope of the line $(6.0 \pm 0.4) \times 10^{4} \mathrm{~s} \mathrm{~cm}^{-2}$, with the values $D_{\mathrm{Ox}}=8.3 \times 10^{-6} \mathrm{~cm}^{2} \mathrm{~s}^{-1}$ and $D_{\mathrm{R}}=1.24 \times 10^{-5} \mathrm{~cm}^{2} \mathrm{~s}^{-1}$ [23], it was calculated that $K=0.61 \pm 0.04$. From the discussion of the critical deposition time this value of $K$ is in reasonably good agreement with the theoretical value of 0.44 in Eqn. 4. This result confirms the quantitative influence of $r_{0}, \bar{\delta}$, and $C_{\mathrm{L}} / C_{\mathrm{M}}$ on the a.s.v. peak parameters, and shows that Eqn. 4 provides an estimate of $C_{\mathrm{M}}^{0}$, even in the presence of a ligand. 
TABLE 2

Values of $t_{\mathrm{c}}$ found for different values of the experimental parameters $r_{0}, \bar{\delta}$ and $C_{\mathrm{L}} / C_{\mathrm{M}}$ (errors were $r_{0}= \pm 0.002 ; \bar{\delta}= \pm 10 \% ; t_{\mathrm{c}}= \pm 12 \mathrm{~s}$ )

\begin{tabular}{llrr}
\hline $\begin{array}{l}r_{0} \\
(\mathrm{~cm})\end{array}$ & $\begin{array}{l}\bar{\delta} \\
(\mathrm{cm})\end{array}$ & $C_{\mathrm{L}} / C_{\mathrm{M}}$ & $\begin{array}{r}t_{\mathrm{c}} \\
(\mathrm{min})\end{array}$ \\
\hline 0.055 & $1.2 \times 10^{-3}$ & 98 & 6.2 \\
0.044 & $1.2 \times 10^{-3}$ & 136 & 7.1 \\
0.044 & $1.2 \times 10^{-3}$ & 98 & 5.2 \\
0.044 & $1.2 \times 10^{-3}$ & 65 & 3.4 \\
0.044 & $1.7 \times 10^{-3}$ & 98 & 7.6 \\
0.044 & $2.3 \times 10^{-3}$ & 98 & 11.0 \\
0.032 & $1.2 \times 10^{-3}$ & 98 & 3.4 \\
\hline
\end{tabular}
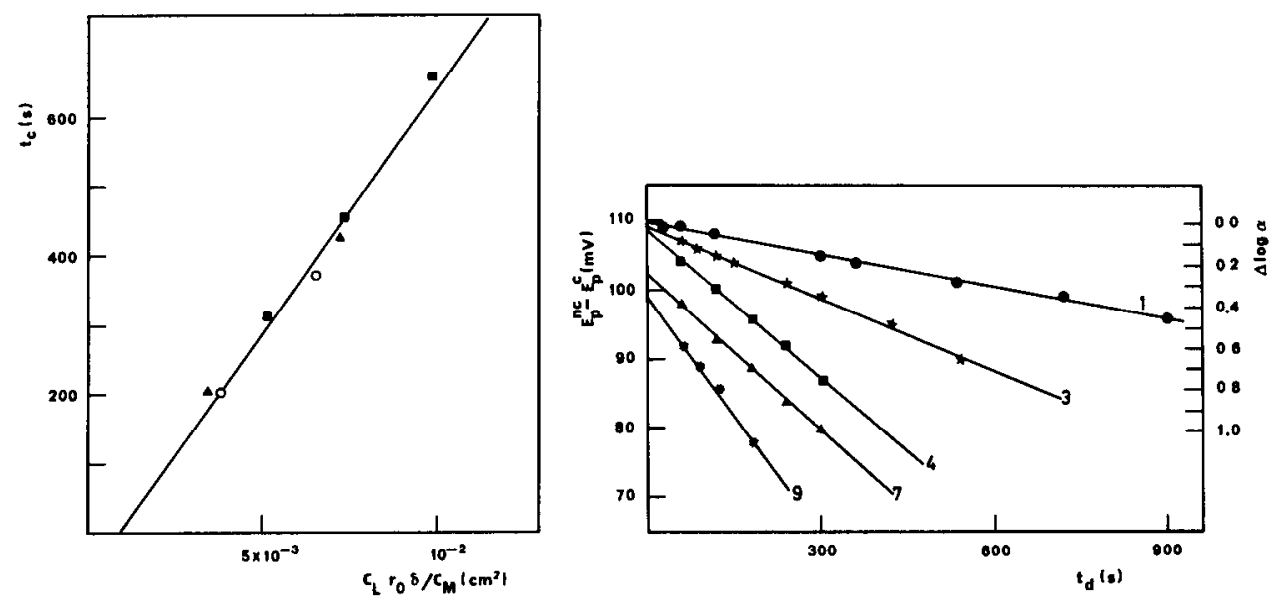

Fig. 7. Influence of the experimental parameters $\bar{\delta}, r_{0}, C_{\mathrm{L}} / C_{\mathrm{M}}$, given in Table 2 , on the critical deposition time $t_{\mathrm{c}}$. (The point at $t_{\mathrm{c}}=11 \mathrm{~min}$ is less reliable, because a very long electrolysis time $(45 \mathrm{~min})$ was used for measurement of $i_{\mathrm{sh}} \cdot(0, \|, \Delta)$ Variations of $r_{0}, \bar{\delta}$, and $C_{\mathrm{L}} / C_{\mathrm{M}}$, respectively (see Table 2 ).

Fig. 8. Change of $E_{\mathrm{p}}^{\mathrm{nc}}-E_{\mathrm{p}}^{\mathrm{c}}$ and $\Delta \log (\alpha)$ with $t_{\mathrm{d}}$, for $t_{\mathrm{d}}<t_{\mathrm{c}}$. Plots correspond to the experimental conditions presented in Table 3 with the same number: (•) $1 ;(\star) 3 ;(\bullet) 4$; (A) $7 ;(*) 9$. The axis $\Delta \log \alpha$ relates to line 3 only.

The $\mathrm{E}_{p}^{c}$ vs. $\mathrm{t}_{d}$ curves, and measurements of $\beta$

Figure 8 shows the change in $E_{\mathrm{p}}^{\mathrm{c}}$ with $t_{\mathrm{d}}$, expressed as $\Delta E_{\mathrm{p}}=E_{\mathrm{p}}^{\mathrm{nc}}-E_{\mathrm{p}}^{\mathrm{c}}$, for $t_{\mathrm{d}}<t_{\mathrm{c}}$. Linear relationships were always obtained within experimental error. This property makes it possible to evaluate $\beta$ by extrapolating $\Delta E_{\mathrm{p}}$ to $t_{\mathrm{d}}=0$ $\left[\Delta E_{\mathrm{p}}(t=0)\right]$, i.e., to conditions where $\alpha_{\mathrm{s}}^{0}=\alpha$. The $\Delta E_{\mathrm{p}}(t=0)$ values were then used with Eqns. 2 and 3 to compute $\beta$. The values obtained in this way for various values of $r_{0}, \bar{\delta}, C_{\mathrm{L}} / C_{\mathrm{M}}$ and $\mathrm{pH}$, are reported in Table 3 . All these values are statistically identical, within experimental error, and the average 


\section{TABLE 3}

Stability constant $(\log \beta)$ evaluated from the intercept of the linear relations $\left(\Delta E_{\mathrm{p}}, t_{\mathrm{d}}\right)$ with slopes $a$, for different values of the experimental parameters $r_{0}, \bar{\delta}, C_{\mathrm{L}}, C_{\mathrm{L}} / C_{\mathrm{M}}$ and $\mathbf{p H}^{\mathbf{a}}$

\begin{tabular}{|c|c|c|c|c|c|c|}
\hline No. & $\begin{array}{l}\bar{\delta} \\
(\mathbf{c m})\end{array}$ & $\begin{array}{l}C_{\mathrm{L}} \\
\left(10^{-4} \mathrm{M}\right)\end{array}$ & $C_{\mathrm{L}} / C_{\mathrm{M}}$ & pH & $\begin{array}{l}\text { Slope } \\
\left(\mu \vee \mathbf{s}^{-1}\right)\end{array}$ & $\log \beta$ \\
\hline 1 & $1.2 \times 10^{-3}$ & 4.90 & 1225 & 7.80 & $15.3 \pm 0.3$ & $10.28 \pm 0.01$ \\
\hline 2 & $1.2 \times 10^{-3}$ & 4.90 & 612 & 7.81 & $23.2 \pm 0.3$ & $10.27 \pm 0.01$ \\
\hline 3 & $1.2 \times 10^{-3}$ & 4.90 & 306 & 7.81 & $34.8 \pm 0.7$ & $10.25 \pm 0.01$ \\
\hline 4 & $1.2 \times 10^{-3}$ & 4.90 & 136 & 7.81 & $72 \pm 2$ & $10.25 \pm 0.01$ \\
\hline 5 & $1.2 \times 10^{-3}$ & 4.90 & 65 & 7.80 & $137 \pm 8$ & $10.21 \pm 0.03$ \\
\hline 6 & $1.2 \times 10^{-3}$ & 0.98 & 98 & 8.05 & $77 \pm 8$ & $10.23 \pm 0.06$ \\
\hline 7 & $1.7 \times 10^{-3}$ & 0.98 & 98 & 8.05 & $76 \pm 2$ & $10.25 \pm 0.01$ \\
\hline 8 & $2.3 \times 10^{-3}$ & 0.98 & 98 & 8.08 & $43 \pm 2$ & $10.27 \pm 0.02$ \\
\hline 9 & $1.2 \times 10^{-3}$ & 0.98 & 98 & 8.02 & $117 \pm 7$ & $10.20 \pm 0.03$ \\
\hline 10 & $1.2 \times 10^{-3}$ & 0.98 & 98 & 8.06 & $88 \pm 5$ & $10.27 \pm 0.03$ \\
\hline
\end{tabular}

ascan rate $20 \mathrm{mV} \mathrm{s}^{-1}, r_{0}=0.044 \mathrm{~cm}$ for all except nos. 9 and 10 , for which $r_{0}=0.032$ and $0.055 \mathrm{~cm}$, respectively.

value, $\log \beta=10.25 \pm 0.03$, is in agreement with that found by differentialpulse cathodic sweep voltammetry (Table 1 ).

It must be pointed out that in the present a.s.v. determination of $\log \beta$, the chief sources of error are those incurred from the measurement of $\Delta E_{\mathrm{p}}(t=0)( \pm 2 \mathrm{mV})$, and $\mathrm{pH}( \pm 0.01)$. These errors produce errors of 0.07 and $0.02 \log$ unit in $\beta$, respectively.

Figure 8 shows that not only does $E_{\mathrm{p}}^{\mathrm{c}}$ increase linearly with $t_{\mathrm{d}}$ (for $t_{\mathrm{d}}<t_{\mathrm{c}}$ ), but that the slopes of the lines depend on the same parameters as $t_{c}$. In this time range, TETA never reaches saturation at the surface, but $\alpha_{\mathbf{s}}^{0}$ decreases gradually when $t_{\mathrm{d}}$ increases. On the basis of this property, it is possible to derive a semiempirical equation (see Appendix) for the change in $\Delta E_{\mathfrak{p}}$ with $t_{\mathrm{d}}$

$\Delta E_{\mathrm{p}}=\Delta E_{\mathrm{p}}(t=0)-\left(1 / K^{\prime}\right)(R T / n F)\left(D_{\mathrm{Ox}} D_{\mathrm{R}}\right)^{1 / 2}\left(C_{\mathrm{M}} / C_{\mathrm{L}}\right) t_{\mathrm{d}} / r_{0} \bar{\delta}$

with $\Delta E_{\mathrm{p}}(t=0)=(R T / n F) \ln \left(\beta C_{\mathrm{L}} / \alpha_{\mathrm{H}}\right) ; K^{\prime}$ is an empirical constant, and should have a value close to 0.44 (Eqn. 4).

The slopes of the straight lines $\Delta E_{\mathrm{p}}$ vs. $t_{\mathrm{d}}$, are given in Table 3 , and are plotted as a function of $C_{\mathrm{M}} /\left(C_{\mathrm{L}} r_{0} \bar{\delta}\right)$ in Fig. 9. A linear relationship is obtained with a slope of $(5.7 \pm 1.8) \times 10^{-4} \mathrm{mV} \mathrm{cm}^{2} \mathrm{~s}^{-1}$. Then by using the above values of $D_{O x}$ and $D_{R}$, the value of $K^{\prime}$ obtained is $0.50 \pm 0.16$.

Effects of medium exchange on the a.s.v. curves

The medium-exchange system was used to verify that changes in the a.s.v. peak parameters with $t_{\mathrm{d}}$, were due to phenomena occurring during the stripping step, and not during the deposition step. 

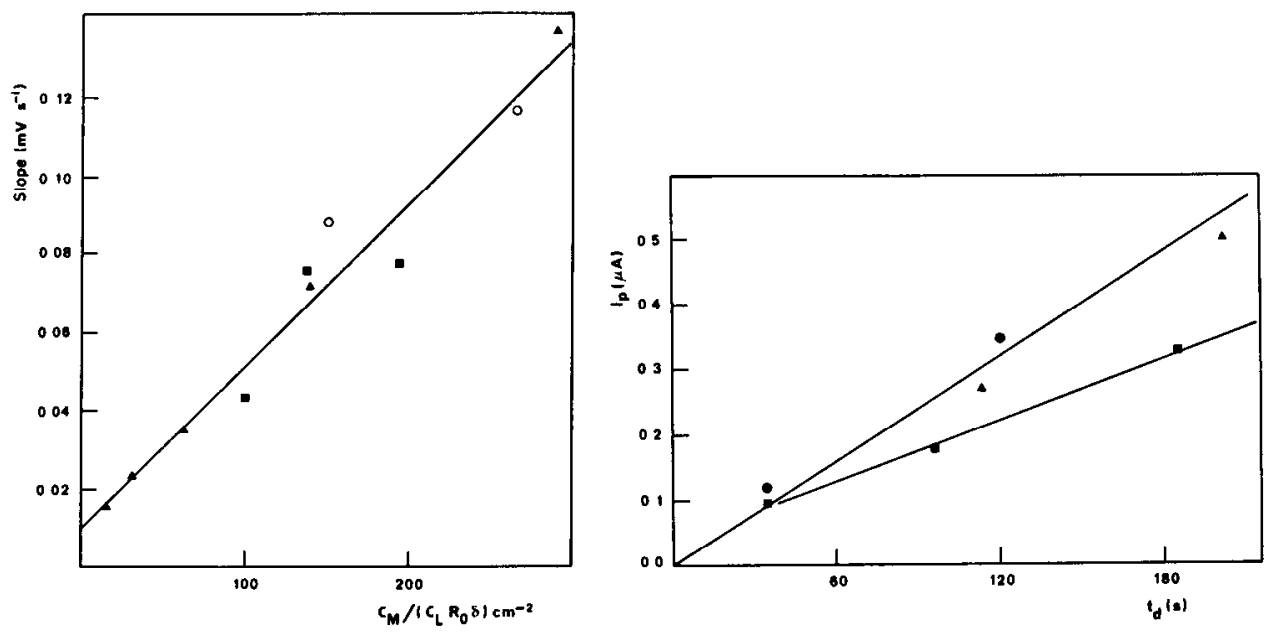

Fig. 9. Influence of the experimental conditions $\bar{\delta}, r_{0}$ and $C_{L} / C_{M}$ on the slopes of the $\Delta E_{\mathrm{p}}$ vs. $t_{\mathrm{d}}$ data. Experimental conditions are given in Table 3 . The symbols $(0,-, \Delta)$ are the same as in Fig. 7.

Fig. 10. Peak current $i_{\mathrm{p}}$ vs. $t_{\mathrm{d}}$ of the a.s.v. curves: $(\bullet)$ in the absence of TETA; $(\bullet)$ in the presence of TETA, without changing the solution; $(\Delta)$ in the presence of TETA, by changing the solution. Each point is the average value of 3-7 determinations. In all cases, experimental errors on $i_{\mathrm{p}}$ varied between 1 and $5 \%$ and $C_{\mathrm{Pb}}=1.0 \times 10^{-6} \mathrm{M}$. For ( $)$ and $(\wedge), C_{\mathrm{L}}=4.6 \times 10^{-5} \mathrm{M}$.

Figure 10 shows $i_{\mathrm{p}}$ vs. $t_{\mathrm{d}}$ relationships in the absence of TETA, and in the presence of TETA without and with medium exchange after the deposition step. It is clear that, without medium exchange, an $i_{\mathrm{p}}$ vs. $t_{\mathrm{d}}$ curve similar to that in Fig. 6 is obtained, whereas, with medium exchange, the points fall on the same straight line as that obtained in the absence of TETA. Not only does this result confirm that the observations described above are due to the stripping step, but also that the diffusion rate of $\mathrm{Pb}^{2+}$ and $\mathrm{PbL}$ during the deposition step are identical.

\section{DISCUSSION}

The results described above show the necessity of considering the concentrations of reagents at the electrode surface when a.s.v. techniques are applied in media containing complexes. The dependences of $i_{\mathrm{p}}$ and $E_{\mathrm{p}}$ on $t_{\mathrm{d}}$ show that the influences of the important factors on $C_{M}^{0}$ are adequately predicted by Eqn. 4 and that this equation can even give a reasonably good estimate of $C_{\mathrm{M}}^{0}$. This is particularly useful, because it allows predictions about the best analytical conditions to be used in order to avoid surface concentration effects with the HMDE. A similar equation is available for mercury film electrodes [5]. Because in many cases $r_{0}, \bar{\delta}, D_{\mathrm{Ox}}$ and $D_{\mathrm{R}}$ do not vary over a 
large range, average values of these parameters $\left(r_{0}=0.04 \mathrm{~cm}, \bar{\delta}=2 \times 10^{-3} \mathrm{~cm}\right.$, $D_{\text {Ox }}=D_{\mathrm{R}}=10^{-5} \mathrm{~cm}$, numerical constant $=0.5$ ) give a useful and widely applicable relationship to calculate the order of magnitude of $C_{\mathrm{M}}^{0} / C_{\mathrm{M}}: C_{\mathrm{M}} / C_{\mathrm{M}}$ $\approx 0.25 t_{\mathrm{d}}(\mathrm{s})$. As discussed above, this makes it possible to calculate that in most complexing media, the ligand/metal ratio in the bulk solution must be larger than 1000 in order to avoid a surface effect.

Figure 6 shows that when this condition is not met, the currents measured are at least $30 \%$ below those that should be obtained in the absence of a surface effect. An even larger discrepancy is obtained when the diffusion coefficient of the complex is lower than that of the free metal ion. This may be important for determinations of total metal concentration when the ligands of the test solution have not been eliminated previously. It may also be very important in attempts to measure the complexing properties of a metal ion in an unknown medium by stripping polarography. Because the importance of this effect will vary along the stripping polarographic wave, it may be expected that not only the limiting current, but also $E_{1 / 2}$ and the Tafel slope could be modified.

\section{Influence on measurements of complex stability constants}

From Fig. 8, it is possible to estimate the error incurred in $\log \alpha$, when the peak potential, $E_{\mathrm{p}}$, is used to compute $\alpha$ from Eqn. 3 without extrapolating its value to $t_{\mathrm{d}}=0$. The value for $\Delta \log \alpha=(n F / R T)\left(\Delta E_{\mathrm{p}}(t=0)-\Delta E_{\mathrm{p}}\right)$ is given in Fig. 8 for curve 3. For example, it can be seen that for $t_{\mathrm{d}}=85 \mathrm{~s}$, an error of $0.1 \log$ unit is made for $\log \alpha$. From all the cases studied here, it can be generalized that $C_{\mathrm{L}} / C_{\mathrm{M}}$ must exceed 1000 if an error smaller than 0.05 log unit is needed. This work suggests that two methods can be used to obtain correct $\alpha$ values, despite the surface effect. First, $\alpha$ can be measured from the shift in $E_{\mathrm{p}}$ extrapolated to $t_{\mathrm{d}}=0$. In cases when $D_{\mathrm{ML}} \neq D_{\mathrm{M}}$, the values of $i_{\mathrm{p}}^{\mathrm{c}}$ and $i_{\mathrm{p}}^{\mathrm{nc}}$ must also be used in Eqn. 3. Because $i_{\mathrm{p}}^{\mathrm{c}}$ is also affected by surface effects, $i_{\mathrm{p}}^{\mathrm{c}} / t_{\mathrm{d}}$ must be extrapolated to $t_{\mathrm{d}}=0$, to give $\left(i_{\mathrm{p}}^{\mathrm{c}} / t_{\mathrm{d}}\right)_{t=0}$. The ratio $\left(i_{\mathrm{p}}^{\mathrm{c}} / t_{\mathrm{d}}\right)_{t=0} /\left(i_{\mathrm{p}}^{\text {nc }} / t_{\mathrm{d}}\right)$ must then be introduced into Eqn. 3 . The second possibility is to use stripping polarography with medium exchange between deposition and stripping. This procedure has the advantage of being potentially applicable not only to labile complexes, as in the preceding case, but to any kind of complexation reaction, because the peak current is then directly proportional to the quantity of metal deposited during the reduction step. The method is, however, much more delicate to apply with the HMDE, especially in preventing penetration of oxygen, and accounting for any reduction during the medium exchange.

\section{Application to measurements of complexation capacity}

Complexation capacity is defined, for natural waters, as the overall concentration of all the complexing sites of the medium able to bind a given metal ion M. It is often obtained by making standard additions of the metal ion to the test medium and measuring the a.s.v. or d.p.a.s.v. peak currents as 
a function of $C_{M}$. Ideally, before the saturation of the ligands, the increase of $i_{\mathrm{p}}$ is smaller than with standard solutions, whereas a linear increase, with the calibration slope, is observed for an excess of metal ion [24]. The value of $C_{\mathrm{M}}$ at the break corresponds to the complexation capacity. This assumes that the complexes formed are electrochemically inert. For labile complexes, the surface concentration effect can simulate, but must not be confused with, a complexation capacity curve [6]. Indeed, because $C_{M}$ and $t_{\mathrm{d}}$ play an equivalent role in Eqn. 4, the curve $i_{\mathrm{p}}=f\left(C_{\mathrm{M}}\right)$ at constant $t_{\mathrm{d}}$ has a similar shape to the $i_{\mathrm{p}}=f\left(t_{\mathrm{d}}\right)$ curve at constant $C_{\mathrm{M}}$ (Fig. 6). Such $i_{\mathrm{p}}$ vs. $C_{\mathrm{M}}$ curves are shown for the Pb/TETA system in Fig. 11, as well as the corresponding $i_{\mathrm{p}}$ vs. $t_{\mathrm{d}}$ curves. The equivalence of $t_{\mathrm{d}}$ and $C_{\mathrm{M}}$ is clearly shown here: for a given value of $C_{\mathrm{L}}$, the change in $i_{\mathrm{p}}$ depends on $t_{\mathrm{d}} C_{\mathrm{M}}$ only if the other experimental conditions remain constant, i.e., $C_{M}$ can be changed by variations in both the deposition time and in the value of $C_{\mathrm{M}}$. A similar curve was reported by Bhat and Weber [13] for the addition of cadmium to fulvic acids, which could be due to the above surface effect. For conditions when $D_{\mathrm{ML}}<D_{\mathrm{M}}$, the "break" in the capacity curve (at $t_{\mathrm{d}}=10 \mathrm{~min}$ in Fig. 6) is even better defined, as $i_{\mathrm{p}}$ decreases in the first part of the curve. This break is related to saturation of the ligand at the electrode surface, but not in the bulk solution. When such a break is interpreted as a bulk complexation capacity, the capacity can be underestimated by a factor of 50-100 [6].

Because it was demonstrated above that $t_{\mathrm{c}}$ is directly proportional to $C_{\mathrm{L}}$ (Eqn. 5), it would be attractive to measure the complexation capacity by titrating the ligand within the diffusion layer by varying $t_{\mathrm{d}}$, instead of by adding metal ion to the bulk solution. This would have the advantage of keeping the value of $C_{\mathrm{L}} / C_{\mathrm{M}}$ constant (and preferably low) in the bulk solu-

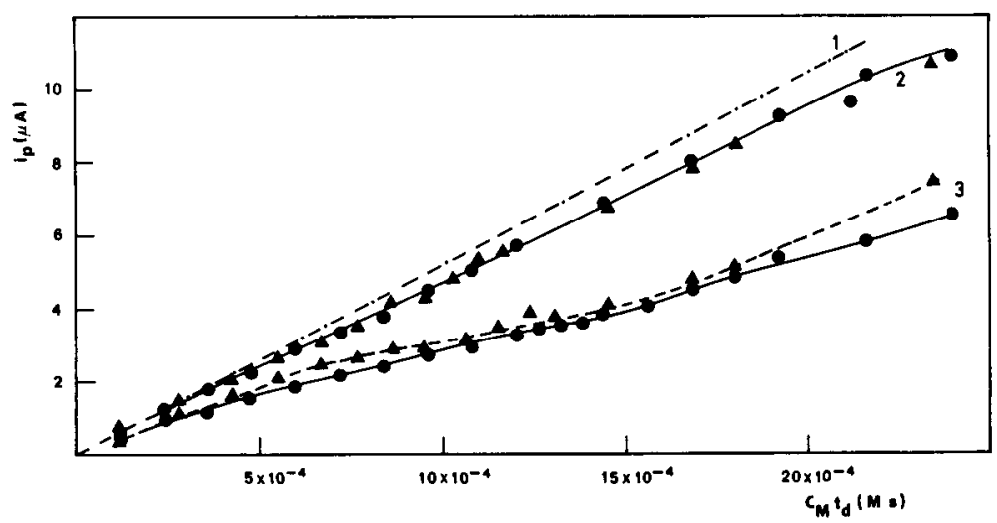

Fig. 11. Change in $i_{\mathrm{p}}$ with $t_{\mathrm{d}}$ and $C_{\mathrm{M}}$. Curves: (1) $C_{\mathrm{L}}=0, \mathrm{pH} 5.5$ (the same curve was obtained for $C_{\mathrm{L}} \rightarrow \infty$ and $\mathrm{pH} 8.0$ ); (2) $C_{\mathrm{L}}=2 \times 10^{-3} \mathrm{M}$ and $3 \times 10^{-2} \mathrm{M}$ triethanolamine at pH 8.00 ; (3) $C_{\mathrm{L}}=4 \times 10^{-4} \mathrm{M}$ and $4 \times 10^{-3} \mathrm{M}$ triethanolamine at pH 8.00. (6) $t_{\mathrm{d}}=2 \mathrm{~min}$ with variable $C_{\mathrm{Pb}} ;(\wedge) C_{\mathrm{Pb}}=2 \times 10^{-6} \mathrm{M}$ with variable $t_{\mathrm{d}}$. In all cases $E_{\mathrm{d}}=-0.750$ and $v=$ $20 \mathrm{mV} \mathrm{s}^{-1}$. 
tion during the whole procedure, hence minimizing possible coagulation or aggregation effects. Despite its apparent elegance, it is doubtful that such a procedure would give accurate results under practical conditions. One reason is that the measurement of $t_{c}$ requires the existence of a sufficiently welldefined shoulder on the a.s.v. peak. This occurs in the case of a very stable "simple" complex like $\mathrm{Pb}$ (II)/TETA, but not with the mixtures of comparatively weak complexes often found in natural waters. Another operational parameter could be used, such as the jump in the $E_{\mathrm{p}}$ vs. $t_{\mathrm{d}}$ curve, but these "characteristic" parameters would be difficult to find in practical media containing mixtures of ligands with different complexation and diffusing properties.

\section{Surface concentration effect in d.p.a.s.v.}

Differential-pulse a.s.v. is more generally used than a.s.v. for application to natural waters, because of its greater sensitivity and its relatively symmetrical peak shapes. Althouth the modulation in potential during the stripping step will produce a variation of the corresponding concentration of metal ion at the surface for the same $t_{\mathrm{d}}$, the order of magnitude of the average value of $C_{M}^{0}$ in d.p.a.s.v. will be the same as for a.s.v. However, because of its much greater sensitivity, a smaller $t_{\mathrm{d}}$ can be used with d.p.a.s.v., thus minimizing the surface concentration effect. For practical reasons, it is difficult to use $t_{\mathrm{d}}<60$ s (i.e., $C_{\mathrm{M}} / C_{\mathrm{M}}<20$ ) whatever the technique, at least with the HMDE. Thus the surface effect is not negligible, even with d.p.a.s.v., and it can be expected to play an important role in any system containing natural ligands.

The authors gratefully acknowledge the technical assistance of $\mathrm{M}$. L. Tercier, F. Bujard and C. Bernard. This work was supported by the Swiss National Foundation (Project 2.413/0.82) and by the Junta Nacional de Investigacao Cientifica e Tecnologica (Contract 315.81.57).

\section{APPENDIX}

During the stripping step, the ligand at the electrode surface becomes more and more saturated as the applied potential, $E$, is scanned to more positive values. This influences the concentration gradients, and in turn, the current and the position of the peak potential. Nevertheless, for $t_{\mathrm{d}}<t_{\mathrm{c}}$ (small decrease in free ligand concentration at the surface compared to the bulk solution, Eqn. 3 can be used as a first approximation by replacing $\alpha$, with its value at the electrode surface, $\alpha_{\mathbf{s}}^{0} . \alpha_{\mathbf{s}}^{0}=1+\beta[L]^{0}$, where $[\mathrm{L}]^{0}$ is the concentration of free $L$ at the surface. Further, neglecting the second term in Eqn. 3 and because $\beta[L]^{0}$ $\gg 1$, gives $\Delta E_{\mathrm{p}} \approx(R T / n F) \ln \left(\beta[\mathrm{L}]^{\circ}\right)$. [L $]^{0}$ must be estimated from the diffusion fluxes of $\mathrm{L}$, its protonated forms and ML at the surface. However, if all these species have similar diffusion coefficients, the approximate mass balance equation at the surface will be $C \mathrm{~L}=$ $C_{\mathrm{L}}=[\mathrm{L}]^{0} \alpha \mathrm{H}_{\mathrm{H}}+[\mathrm{ML}]^{0}$, and $C_{\mathrm{M}}=[\mathrm{M}]^{0}+[\mathrm{ML}]^{0}$. Because the system is well buffered, $\alpha_{\mathbf{H}} \approx \alpha_{\mathrm{H}}$. Under the conditions used here, $L$ is not saturated and the ML complexes are very stable, so that $C_{M}^{0} \approx[M L]^{0}$. Finally, $C_{M}^{0}$ is evaluated from Eqn. 4 , where the numerical constant is replaced by $K^{\prime}$. Combining these conditions with the above equations for $\Delta E_{\mathrm{p}}, C_{\mathrm{L}}^{\mathrm{L}}$ and $C_{\mathrm{M}}$ yields 
$\Delta E_{\mathrm{p}}=(R T / n F)\left\{\ln \left(\beta C_{\mathrm{L}} / \alpha_{\mathrm{H}}\right)+\ln \left[1-\left(1 / K^{\prime}\right)\left(D_{\mathrm{Ox}} / D_{\mathrm{R}}\right)^{1 / 2}\left(C_{\mathrm{M}} / C_{\mathrm{L}}\right) t_{\mathrm{d}} / r_{\mathrm{o}} \bar{\delta}\right]\right\}$

For $t_{\mathrm{d}}<\left[r_{0} \bar{\delta} /\left(D_{\mathrm{Ox}} D_{\mathrm{R}}\right)^{1 / 2}\right]\left(C_{\mathrm{L}} / C_{\mathrm{M}}\right)$. Eqn. A1 reduces to a linear relationship

$\Delta E_{\mathrm{p}}=(R T / n F)\left\{\ln \left(\beta C_{\mathrm{L}} / \alpha_{\mathrm{H}}\right)-\left(1 / K^{\prime}\right)(R T / n F)\left(D_{\mathrm{Ox}} D_{\mathrm{R}}\right)^{1 / 2}\left(C_{\mathrm{M}} / C_{\mathrm{L}}\right) t_{\mathrm{d}} / r_{0} \bar{\delta}\right\}$

The $\Delta E_{\mathrm{p}}$ values were computed from both Eqns. A1 and A2 with the values of $K^{\prime}, r_{0}, \bar{\delta}$, $D_{\mathrm{Ox}}$ and $D_{\mathrm{R}}$ found in this work, and using values of $C_{\mathrm{L}} / C_{\mathrm{M}}=50,100$ and 300 . It was found that for the difference in the two $\Delta E_{\mathrm{p}}$ values to be less than $2 \mathrm{mV}$ (i.e., to achieve a linear dependence of $E_{\mathrm{p}}$ vs. $t_{\mathrm{d}}$, the $t_{\mathrm{d}}$ values had to be less than 1,2 and $6 \mathrm{~min}$, respectively. In practically similar conditions, the $E_{\mathrm{p}}$ vs. $t_{\mathrm{d}}$ curves were found to be linear over a larger range of $t_{\mathrm{d}}$, a result that may well be due to the approximate nature of the above mathematical treatment.

\section{REFERENCES}

1 A. Zirino, in M. Whitfield and D. Jagner (Eds.), Marine Electrochemistry, Wiley, Chichester, 1981, Ch. 10.

2 H. W. Nurnberg, in P. Ahlberg and L. O. Sundelof (Eds.), Structure and Dynamics in Chemistry, 1978, p. 270.

3 W. Davison and M. Whitfield, J. Electroanal. Chem., 75 (1977) 763.

4 J. E. Spell II and R. H. Philp Jr, J. Electroanal. Chem., 12 (1980) 281.

5 J. Buffle, J. Electroanal. Chem., 125 (1981) 273.

$6 \mathrm{~J}$. Buffle, A. Tessier and W. Haerdi, in C. J. Kramer and J. C. Duinker (Eds.), Complexation of Trace Metals in Natural Waters, Martinus Nijhoff, D. W. Junk Publishers, The Hague, 1984, p. 301.

$7 \mathrm{~J}$. Buffle, in H. Sigel (Ed.), Metal ions in Biological Systems, Vol. 18, M. Dekker, New York, 1984, Ch. 6.

8 H. Bilinski, R. Huston and W. Stumm, Anal. Chim. Acta, 84 (1976) 157.

9 H. W. Nurnberg, P. Valenta, L. Mart, B. Raspor and L. Sipos, Z. Anal. Chem., 282 (1976) 357.

10 M. Branica, D. M. Novak and S. Bubic, Croat. Chem. Acta, 49 (1977) 539.

11 A. Zirino and S. P. Kounaves, Anal. Chem., 49 (1976) 56.

12 M. S. Shuman and J. L. Cromer, Anal. Chem., 51 (1979) 1546.

13 G. A. Bhat and J. H. Weber, Anal. Chim. Acta, 41 (1982) 95.

14 I. Ružić, Anal. Chim. Acta, 140 (1982) 99.

15 M. S. Shuman and G. P. Woodward, Environ. Sci. Technol., 11 (1977) 809.

16 R. M. Smith and A. E. Martell, Critical Stability Constants, Vol. 2, Plenum Press, New York, 1975.

17 W. Davison, J. Electroanal. Chem., 87 (1978) 395.

$18 \mathrm{M}$. V. Stackelberg, M. Pilgram and V. Toome, Z. Electrochem., 57 (1953) 342.

19 E. R. Brown and R. F. Large, in A. Weissberger (Ed.), Techniques of Chemistry, Vol. I,

Part 2a, Wiley-Interscience, New York, 1971, Ch. 6.

20 A. Ringbom, Complexation in Analytical Chemistry, Interscience, New York, 1963.

21 D. D. DeFord and D. N. Hume, J. Am. Chem. Soc., 73 (1951) 5321.

$22 \mathrm{~J}$. Heyrovsky and J. Kuta, Principles of Polarography, Academic Press, New York, 1966, Ch. 8 and 12.

23 Z. Galus, Diffusion Coefficients of Metals in Mercury, Pure Appl. Chem., 56 (1984) 635.

24 Y. K. Chau and K. Lum Shue Chan, Water Res., 8 (1974) 383. 